Prel i minary i nvesti gati on of the cl i ni cal usef ul ness of super-hi gh-resol ut i on LCDs wi th 9 and 15 mega sub- pi xel s: Observati on studi es wi th phant ons

\begin{tabular}{|l|l|}
\hline 著者 & $\begin{array}{l}\text { N shi mur a Aya, I chi kawa Kat suhi ro, Mochi ya } \\
\text { Yuko, Mor i shi ta Ayum, Kawashi ma H r oko, } \\
\text { Yamannt o Tomoyuki, Hasegawa M ki o, K mur a } \\
\text { Naof um, Sanada Shi ger u }\end{array}$ \\
\hline $\begin{array}{l}\text { j our nal or } \\
\text { publ i cat i on ti t l e }\end{array}$ & Radi ol ogi cal Physi cs and Technol ogy \\
\hline vol une & 3 \\
\hline number & 1 \\
\hline page r ange & $70-77$ \\
\hline year & $2010-01-01$ \\
\hline URL & ht t p: //hdl . handl e. net /2297/23497 \\
\hline
\end{tabular}




\title{
Preliminary investigation of the clinical usefulness of super-high-resolution LCDs with 9 and 15 mega-sub-pixels: observation studies with phantoms
}

\author{
Aya Nishimura $\cdot$ Katsuhiro Ichikawa $\cdot$ Yuko Mochiya $\cdot$ Ayumi Morishita $\cdot$ \\ Hiroko Kawashima • Tomoyuki Yamamoto • Mikio Hasegawa • \\ Naofumi Kimura $\cdot$ Shigeru Sanada
}

Received: 30 August 2009/Revised: 27 November 2009/Accepted: 30 November 2009/Published online: 25 December 2009

(C) Japanese Society of Radiological Technology and Japan Society of Medical Physics 2009

\begin{abstract}
Our purpose in this study was to evaluate the preliminary clinical efficacy of soft-copy reading of digital mammography, for a 15-mega-sub-pixel (MsP) and a 9-MsP super-high-resolution liquid-crystal display (SHRLCD) by use of an independent sub-pixel driving technology. We performed three kinds of phantom observation studies by six radiological technologists. Detectability of a contrast-detail phantom and simulated small objects (SSOs) resembling microcalcifications (MCLs), and shape discrimination ability of SSOs with round and square shapes, were examined and compared with a 5-MP conventional LCD (5-MP LCD). In each study, four types of display magnification ratio were used. The detectability and the shape discrimination ability of the 15-MsP SHRLCD were highest among the three LCDs of most of the display magnification ratios. The 9-MsP SHR-LCD indicated a higher or equal performance as compared with the 5-MP LCD in the SSO detection and shape studies. The results of our study demonstrated that the SHR-LCDs had good potential to detect MCLs and to evaluate the shape in high-resolution digital mammography.
\end{abstract}

A. Nishimura $(\bowtie) \cdot$ Y. Mochiya $\cdot$ A. Morishita $\cdot$ T. Yamamoto Department of Radiology, Kanazawa University Hospital, 13-1 Takaramachi, Kanazawa 920-8641, Japan

e-mail: aya_nsmr@med.kanazawa-u.ac.jp

K. Ichikawa $\cdot$ H. Kawashima $\cdot$ S. Sanada

Graduate School of Medical Science, Kanazawa University,

5-11-80 Kodatsuno, Kanazawa 920-0942, Japan

M. Hasegawa · N. Kimura

Totoku Electric Co., Ltd, 300 Oya, Ueda,

Nagano 386-0192, Japan
Keywords Liquid-crystal display (LCD) · Digital mammography $\cdot$ Sub-pixel $\cdot$ Independent sub-pixel driving (ISD)

\section{Introduction}

Diagnostic soft-copy reading on liquid-crystal displays (LCDs) is becoming widespread in medical fields with the increasing utilization of digital imaging modalities. Highresolution LCDs, such as 3-megapixel (MP) and 5-MP LCDs, are currently recommended for soft-copy reading in digital mammography [1-3]. However, flat-panel detectors (FPDs) and computed radiography (CR) systems for digital mammography have already achieved resolutions in the range of 13-65 MP, and even 5-MP LCDs, the highestresolution display available, do not have sufficient resolution for FPD and CR systems. Therefore, LCDs with higher resolution are required for use in diagnostic soft-copy reading in mammography, which requires detailed interpretation of very fine lesions [3-5].

Currently, super-high-resolution LCDs (SHR-LCDs) with a new resolution-enhancement technology named independent sub-pixel driving (ISD) are being developed, and their clinical efficacy is expected in various medical fields. The technology enhances the resolution of LCDs by threefold by utilizing three sub-pixels included in each pixel element of the LCDs for image rendering. By implementation of this technology in conventional monochrome medical LCDs, 15-mega-sub-pixel (MsP) and 9-MsP SHR-LCDs were developed from conventional monochrome 5-MP and 3-MP LCDs, respectively [4, 5]. Figure 1 shows a comparison of an image displayed on a conventional 5-MP LCD and that on the enhanced 15-MsP SHR-LCD. The figures show curved lines with a fine 
Fig. 1 Displayed image of curved lines with fine horizontal pitch on the conventional 5-MP LCD and 15-MsP SHR-LCD with ISD technology. The capture areas were approximately $25 \times 25 \mathrm{~mm}^{2}$
$5 \mathrm{M}$

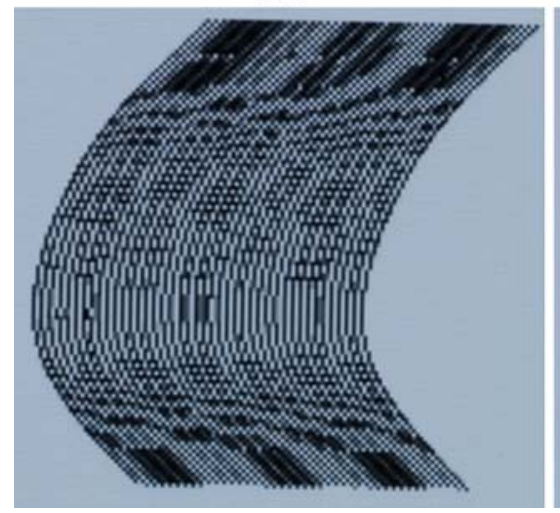

$15 \mathrm{M}$

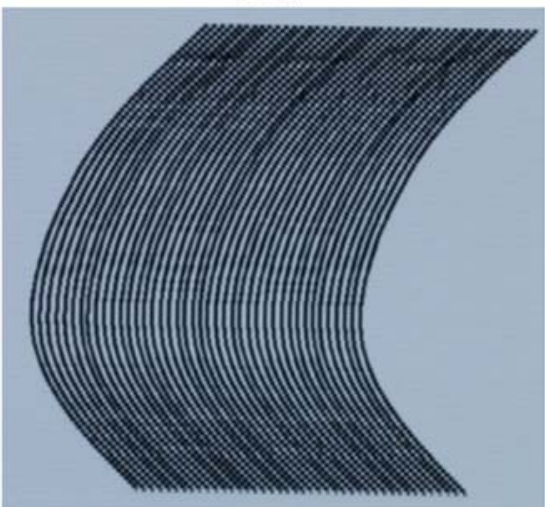

horizontal pitch; the lines could be clearly distinguished on the 15-MsP SHR-LCD because of its higher resolution.

Several recent studies reported the clinical efficacy of conventional 3-MP and 5-MP LCDs, such as detectability and display properties [1-3, 6, 7]. For the SHR-LCD, Shiraishi et al. [8] reported on an observer study evaluation comparing a 9-MsP SHR-LCD with a conventional 3-MP LCD by use of digital mammography. They concluded that the 9-MsP LCD has the potential to increase the sensitivity in the detection of microcalcifications (MCLs), and to improve the visibility of MCLs, especially when highresolution digital mammograms are displayed. However, 5-MP LCDs are recommended for reading of digital mammograms in many countries, such as the USA, Japan, and many European countries. Therefore, a comparative study of the SHR-LCD with the 5-MP LCD is required for evaluation of the usefulness of the SHR-LCD.

As radiologists must detect the MCLs and evaluate their characteristics, such as shape and distribution type, in diagnostic reading of digital mammograms, it is expected that higher-resolution LCDs will provide better detectability and shape discrimination ability for the MCLs. The SHR-LCDs achieve a threefold higher-resolution than do current LCDs in only the sub-pixel direction $[4,5,9,10]$. Therefore, as the SHR-LCDs provide higher resolution only in the sub-pixel direction, it is necessary to carefully investigate their effectiveness for displaying digital mammograms.

As a preliminary step to observational studies using clinical digital mammograms, we performed two types of phantom study to evaluate the efficacy of 9- and 15-MsP SHR-LCDs as compared with 5-MP LCDs. The observers were six radiologic technologists who had sufficient experience with clinical mammographic examinations. We employed a contrast-detail phantom which is generally used for mammographic image quality evaluation, as well as computer-simulated small objects with round or square shapes implanted in a noise image. The performance of the SHR-LCDs was evaluated by comparison of the detectabilities and shape discrimination abilities for the 15- and 9-MsP SHR-LCDs with those of the conventional 5-MP LCD.

\section{Materials and methods}

\subsection{Display systems and viewing conditions}

We employed a conventional 5-MP LCD (ME551i; Totoku, Tokyo, Japan) and two SHR-LCDs with resolutions of 9- and 15-MsP. The 9- and 15-MsP SHR-LCDs were developed from 3- and 5-MP monochrome LCDs (ME551i and ME351i; Totoku), respectively, by equipping them with ISD electric circuits and dedicated firmware.

To differentiate the size of an ordinary pixel from that of one sub-pixel which was treated as one pixel in the SHRLCDs, we defined the size of one sub-pixel in the sub-pixel chain direction as "sub-pixel size," and the size of one pixel on the conventional LCD and of one sub-pixel in the direction perpendicular to the sub-pixel chain direction as "normal pixel size."

The normal pixel sizes of the 5-MP LCD, 15-MsP SHR-LCD, and 9-MsP SHR-LCD (5-MP, 15-MsP, and 9-MsP, respectively) were $0.165,0.165$, and $0.207 \mathrm{~mm}$, respectively. The sub-pixel sizes of $15-\mathrm{MsP}$ and $9-\mathrm{MsP}$ were 0.055 and $0.069 \mathrm{~mm}$, respectively. The maximum luminance values of all LCDs were adjusted to $500 \mathrm{~cd} / \mathrm{m}^{2}$. The grayscale functions were calibrated to the grayscale standard display function (GSDF) defined in Digital Imaging and Communications in Medicine (DICOM): Part $14[11,12]$.

The observers in this study were six radiologic technologists who had more than two years of clinical experience in mammography. Three of the observers each had a certificate reading mammograms from the Central Committee on Quality Control of Mammographic Screening in Japan. The windowing condition for display and the distance from the display surface in the observation were 
freely set by the observers. The room illuminance was set to approximately 50 lux, as recommended by the above committee.

\subsection{Obtaining the phantom image and image display on LCDs}

A clinical full-field digital mammography system (Mammomat Novation DR; Siemens, Munich, Germany) was used for acquisition of phantom images. This system had an amorphous selenium direct flat-panel detector with a pixel size of $0.07 \mathrm{~mm}$ and a matrix size of $2560 \times 3328$ for a $180 \times 240 \mathrm{~mm}^{2}$ field. As the objects for imaging, a CDMAM phantom type 3.4 (Nuclear Associates, Venlo, The Netherlands) and acrylic plates were employed.

When the CDMAM was exposed, the phantom was sandwiched between a 10-mm (upside) and two 10-mm (20-mm, downside) acrylic plates. The X-ray exposure conditions were selected as $28 \mathrm{kV}, 50 \mathrm{mAs}, \mathrm{Mo} / \mathrm{Mo}$ filter, approximately same amount of image noise as clinical mammograms could be presented in the phantom image.

The original noise image for the computer-simulated small objects was obtained by use of an acrylic plate with 30 -mm thickness uniformly exposed to X-rays $(28 \mathrm{kV}, 50$ $\mathrm{mAs}$, Mo/Mo filter). The images obtained were displayed at four display magnification levels on the respective LCDs, i.e., $43,55,73$, and $100 \%$, which were defined as the sub-sampling ratios in the normal pixel direction for 5-MP and 15-MsP. Therefore, the mammograms were displayed in the same size on both 5-MP and 15-MsP. At these magnification ratios, $100 \%$ indicates a pixel-by-pixel display, and $43 \%$ indicates a real size display. A ratio of $55 \%$ was used in the primary clinical reading, which the observers have used routinely in a clinical setting. As much as $73 \%$ was selected as a value intermediate between $55 \%$ and $100 \%$. The ratios for $9-\mathrm{MsP}$ were adjusted to yield displayed images of the same size as for 5-MP and 15-MsP. Therefore, the determined magnification ratios for 9-MsP were $34,43,58$, and $79 \%$, corresponding to the four ratios for 5-MP.

\subsection{Observational study using the CDMAM phantom}

The CDMAM phantom was designed for image quality evaluation in mammography systems. It consists of square cells organized in 16 rows and 16 columns. Each cell has one disk at the center, with another positioned in a randomly selected corner. The disk diameter is scaled logarithmically from 0.06 to $2.00 \mathrm{~mm}$, and the disk thickness is scaled logarithmically from 0.03 to $2.0 \mu \mathrm{m}$ (Fig. 2) [3, 13-15].

The six observers independently evaluated the entire images under 12 display conditions, the four display

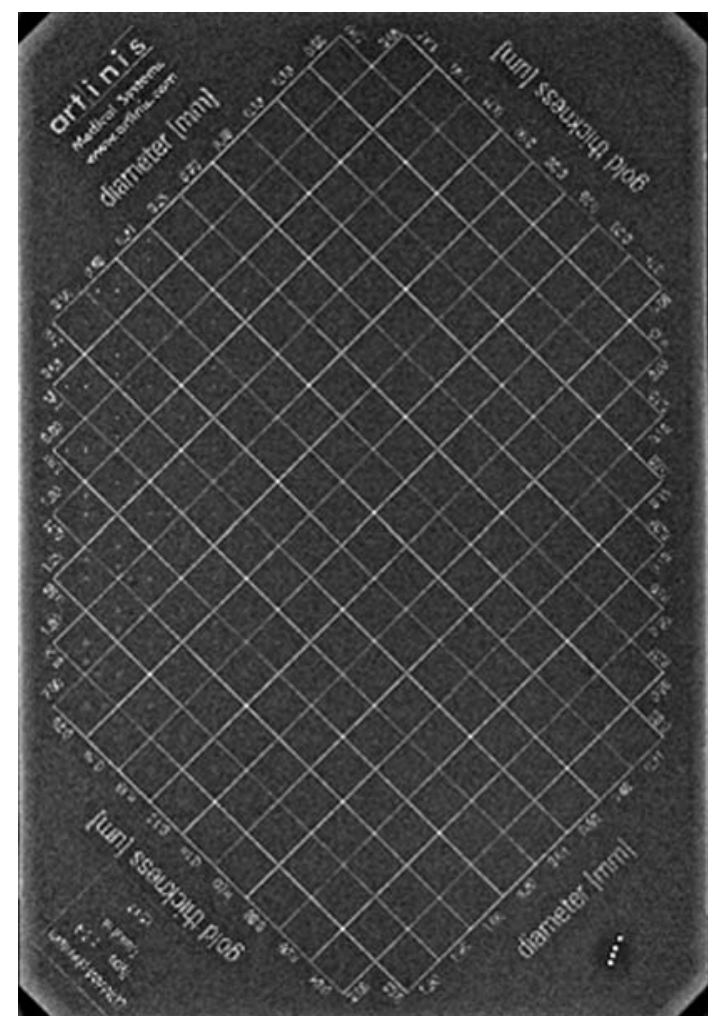

Fig. 2 Sample image of the CDMAM-phantom, type 3.4

magnification ratios for the three LCDs. All observers had sufficient reading training before actual evaluation. All observers were required to identify the location of the corner disks in each square cell region. The observation time per image was not restricted.

Based on the recorded observer responses, a minimum threshold diameter of accurately detected disks was obtained for each different thickness as a measure of contrast in regions of valid detection. The contrast-detail curve ( $\mathrm{C}-\mathrm{D}$ curve) is a graphic correlation between the minimum correct reading diameter and the thickness $[13,14]$; the lower the curve appears on the plot, the better the image quality of the display. In this study, we averaged the C-D curves for the six observers, and we compared them among the three LCDs for each display magnification.

The image quality figure (IQF) is the sum of the products of the minimum diameter and relative thickness. In this study, we used the inverse IQF $\left(\mathrm{IQF}_{\text {inv }}\right)$, determined as follows:

$\mathrm{IQF}_{\mathrm{inv}}=\frac{n}{\sum_{i=1}^{n} D_{i} \times C_{i}, \min }$,

where $\mathrm{C}_{i}$ represents the thickness $(\mu \mathrm{m})$ of column $i$ and $\mathrm{D}_{i}$ denotes the threshold diameter $(\mathrm{mm})$ in contrast column $i$. The parameter $n$ represents the number of rows/columns. In this equation, a higher $\mathrm{IQF}_{\text {inv }}$ value is associated with higher detectability. We averaged the $\mathrm{IQF}_{\text {inv }}$ values for 
the six observers at each magnification ratio and compared them among the three LCDs.

\subsection{Detection and shape discrimination study using simulated small objects}

The CDMAM phantom has only one cell for each combination of diameter and thickness. Therefore, as the appearance of a small disk is often influenced by statistical changes in noise conditions at the position of the disk, the readability of the disk is also influenced by these conditions. In addition, non-uniformity of the obtained image may have an influence. Thus, for obtaining the correct results, the acquisition of more images with different exposures and larger numbers of observations are effective in improving the accuracy. However, in the CDMAM reading task, it is laborious for the observer to evaluate many signals per CDMAM phantom image. Accordingly, it was difficult to obtain stable and correct results in the CDMAM reading study.

We carried out two additional detection studies by using computer-simulated small-object data (SSO) resembling the MCLs. One was a detection study using a round-shaped SSO (SSO detection study), intended for obtaining more accurate detectability results and verifying the results of the CDMAM phantom study. The other was a shape discrimination study in which we used small round and square objects (SSO shape study). For diagnosis of breast cancer, radiologists have attempted to define criteria to pinpoint suspicious lesions and assist in the evaluation of MCLs by using various properties, such as the shape, size, location, and density of MCLs [16, 17]. Because shape evaluation is very important in mammography reading, we added this shape discrimination study for evaluating the display quality of the LCDs.

The common methodology in the two procedures was based on observation of a simulated small object added numerically into the actual noise image obtained from a uniform acrylic phantom, as mentioned in Sect. 2.3. For obtaining more realistic images, the SSOs were processed with a blurring function measured from the mammography system used in this study, and then added numerically to the noise image at a specified position. The digital contrast value relative to the background and size of the object were given arbitrarily during the object creation process.

\subsubsection{SSO detection study}

In the SSO detection study, the observer identified a single SSO placed at a random position in the noise image. As the $\mathrm{SSO}$, a round dot with a diameter of $0.15 \mathrm{~mm}$ and a digital contrast value of 150 relative to the background was used. We used dedicated custom software to create and display

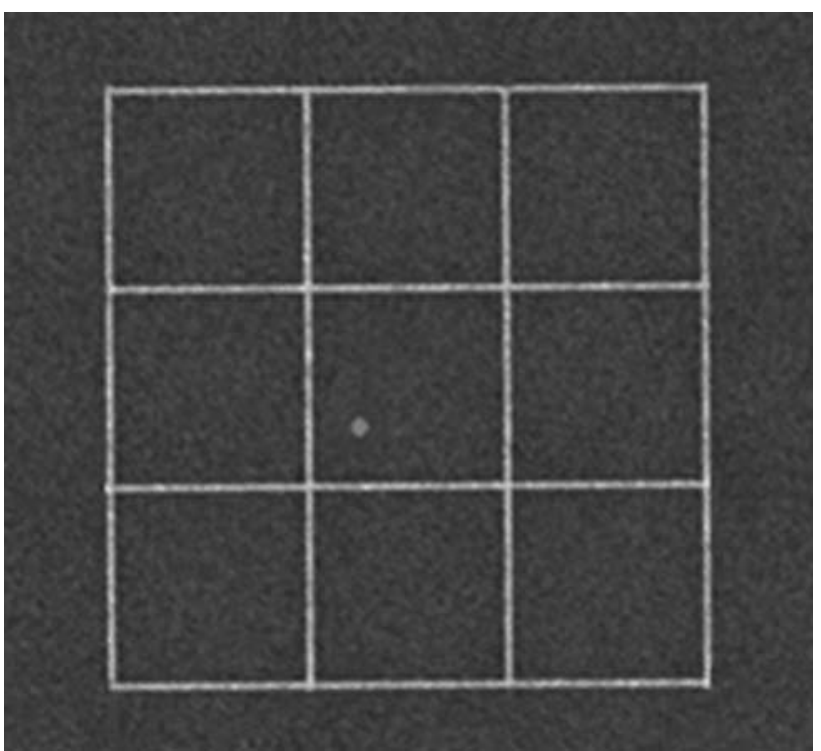

Fig. 3 Noise image on which nine square cells (each cell measuring $30 \mathrm{~mm} \times 30 \mathrm{~mm}$ ) were drawn. A single round small object was implanted in one of the nine cells at a random position within the cell

the simulated images with the specified magnification ratios. The software drew the nine square cells with each cell of $30 \mathrm{~mm} \times 30 \mathrm{~mm}$ at the center of the noise image, and implanted the object at a random position within a cell which was randomly selected (Fig. 3). The observer read 100 images for each magnification ratio. The object appeared for $5 \mathrm{~s}$ per image. During this time, the observer indicated the correct position of the object by clicking with a computer mouse. After $5 \mathrm{~s}$ without mouse clicking, the image was changed after displaying a blank image for $1 \mathrm{~s}$.

The diameter and contrast of the simulated round object were selected in preliminary studies to provide a detectability of $35 \%$ to $70 \%$. We defined the detectability as the correct answer rate among 100 readings. The detectability results for all observers were averaged for each magnification ratio. Each observer underwent sufficient training before the actual study.

\subsubsection{SSO shape study}

Another study was performed for discriminating the shapes (round or square) of the SSOs in the display. The square shape measured $0.31 \mathrm{~mm} \times 0.31 \mathrm{~mm}$, and the diameter of the round shape was set to $0.35 \mathrm{~mm}$; therefore, the two shapes had the same area. A digital contrast value of 150 was used for the two shapes. The dedicated software implanted the object at a random position within a part of the noise image with an area of $90 \mathrm{~mm} \times 90 \mathrm{~mm}$. The reading time per appearance of the object was not restricted in this discrimination study. Each observer read 100 images for each magnification ratio. Shape discrimination ability 
Fig. 4 Comparison of the averaged $\mathrm{C}-\mathrm{D}$ curves of the respective LCDs with the four types of display magnification ratio of a $43 \%$, b $55 \%$, c $73 \%$, and d $100 \%$
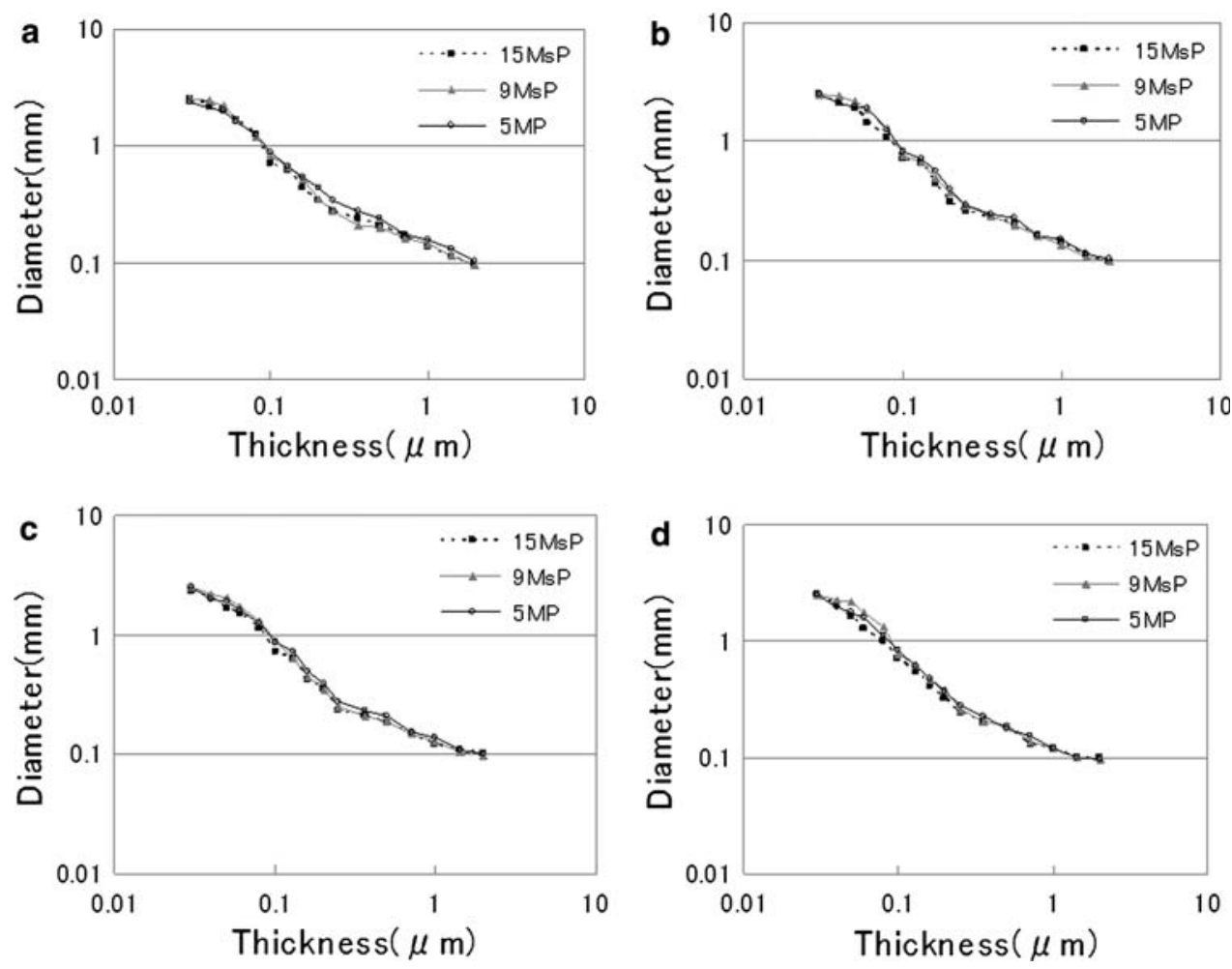

was defined as the correct answer rate among 100 readings. The observer performed this evaluation for twelve conditions, i.e., three LCDs with four display magnification ratios.

\subsection{Statistical analysis}

We performed three comparisons: $15 \mathrm{MsP}$ vs. $9 \mathrm{MsP}$, $15 \mathrm{MsP}$ vs. $5 \mathrm{MP}$, and $9 \mathrm{MsP}$ vs. $5 \mathrm{MP}$. To test the statistical significance of the difference between each LCD pair, we performed a nonparametric test (the Wilcoxon test). The reason for using the Wilcoxon test was that we had only six observers, and this distribution of differences between each pair was non-normally distributed. For each LCD pair, the statistical significance was estimated by use of a $p$-value generated by the Wilcoxon test. A $p$-value of $<0.05$ was considered to indicate a statistically significant difference.

\section{Results}

\subsection{CDMAM phantom study}

The averaged C-D curves for the three LCDs with the four types of display magnification ratio ((a) $43 \%$, (b) $55 \%$, (c) $73 \%$, (d) 100\%) are shown in Fig. 4. The minimum diameters on 15-MsP and 9-MsP indicated lower values

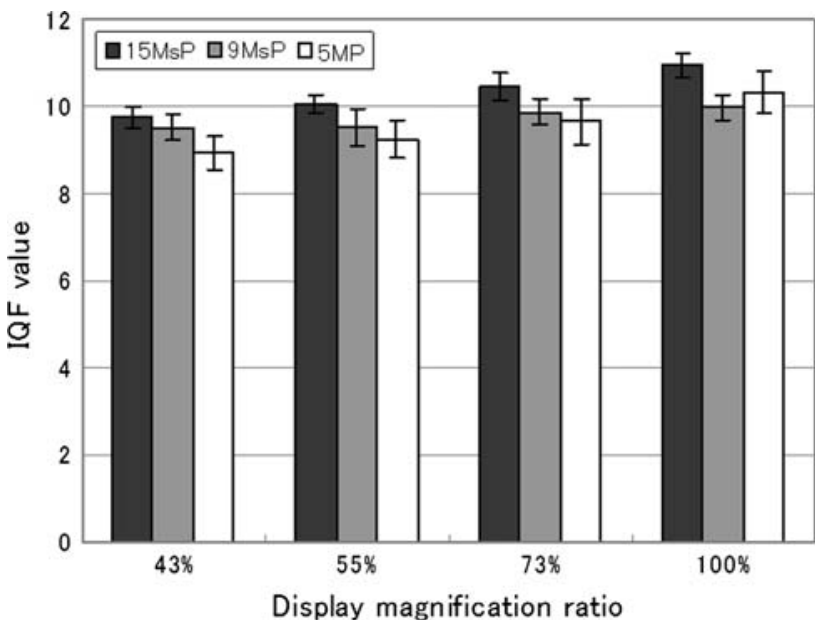

Fig. 5 Comparison of averaged $\mathrm{IQF}_{\text {inv }}$ values for 15-MsP, 9-MsP, and 5-MP with the four types of display magnification ratio

than 5-MP for the middle thickness at the $43 \%$ magnification. At the other magnification ratios, 15-MsP and 9MsP showed almost the same values, and they provided slightly lower values than did 5-MP.

The averaged $\mathrm{IQF}_{\text {inv }}$ values for the three LCDs with the four types of display magnification ratios are shown in Fig. 5, and the results of statistical analysis for each display pair (15 MsP vs. $9 \mathrm{MsP}, 15 \mathrm{MsP}$ vs. $5 \mathrm{MP}$, and $9 \mathrm{MsP}$ vs. $5 \mathrm{MP}$ ) are shown in Table 1 . The averaged $\mathrm{IQF}_{\text {inv }}$ values for the 15-MsP with the four magnification ratios were 
Table 1 Results of statistical analysis of $\mathrm{IQF}_{\text {inv }}$ values for each LCD pair with the four types of display magnification ratio

\begin{tabular}{lllll}
\hline & $p$-value & & \\
\cline { 2 - 5 } & $43 \%$ & $55 \%$ & $73 \%$ & $100 \%$ \\
\hline $15 \mathrm{MsP}$ vs. 9 MsP & 0.3454 & 0.1159 & $0.0277^{*}$ & $0.0277^{*}$ \\
$15 \mathrm{MsP}$ vs. 5 MP & $0.0277^{*}$ & $0.0464 *$ & 0.0747 & $0.0464 *$ \\
$9 \mathrm{MsP}$ vs. 5 MP & 0.0747 & 0.2489 & 0.4631 & 0.3454
\end{tabular}

$* p<0.05$

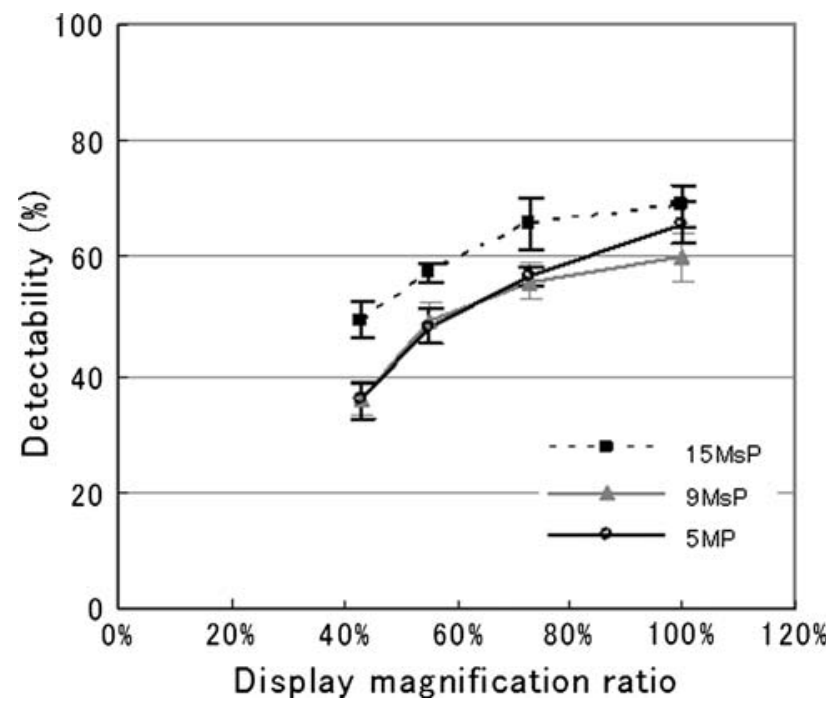

Fig. 6 Comparison of averaged SSO detectability results for the three LCDs with the four types of display magnification ratio

9.76, 10.06, 10.46, and 10.96, respectively. The detectability of $15-\mathrm{MsP}$ was the highest among the three LCDs at all display magnification ratios. At magnification ratios of 43, 55, and $100 \%$, the averaged $\mathrm{IQF}_{\text {inv }}$ values for the $15-\mathrm{MsP}$ were higher than those for 5-MP. These differences were statistically significant $(p=0.0277,0.0464$, and 0.0464 , respectively). The averaged $\mathrm{IQF}_{\text {inv }}$ values for the 9-MsP were positioned between 5-MP and 15-MsP except at the $100 \%$ magnification ratio. The averaged $\mathrm{IQF}_{\text {inv }}$ values for the 9-MsP were higher than those for 5-MP; these differences were not statistically significant $(p>0.0747)$.

\subsection{SSO detection study}

The averaged SSO detectabilities for the three LCDs with the four types of display magnification ratio are shown in Fig. 6, and the results of the statistical analysis for each display pair are shown in Table 2. The averaged SSO detectability values for the 15 -MsP with the four magnification ratios were $49.33,57.50,65.67$, and 68.83 , respectively. The detection ratio of $15-\mathrm{MsP}$ was the highest
Table 2 Results of statistical analysis of SSO detectability for each LCD pair with the four types of display magnification ratio

\begin{tabular}{lllll}
\hline & $p$-value & & \\
\cline { 2 - 5 } & $43 \%$ & $55 \%$ & $73 \%$ & $100 \%$ \\
\hline $15 \mathrm{MsP}$ vs. 9 MsP & $0.0273^{*}$ & $0.0350^{*}$ & $0.0277^{*}$ & $0.0464 *$ \\
$15 \mathrm{MsP}$ vs. 5 MP & $0.0273^{*}$ & $0.0273^{*}$ & $0.0452^{*}$ & 0.0578 \\
$9 \mathrm{MsP}$ vs. 5 MP & 0.9163 & 0.7518 & 0.7512 & 0.0740 \\
\hline
\end{tabular}

$* p<0.05$

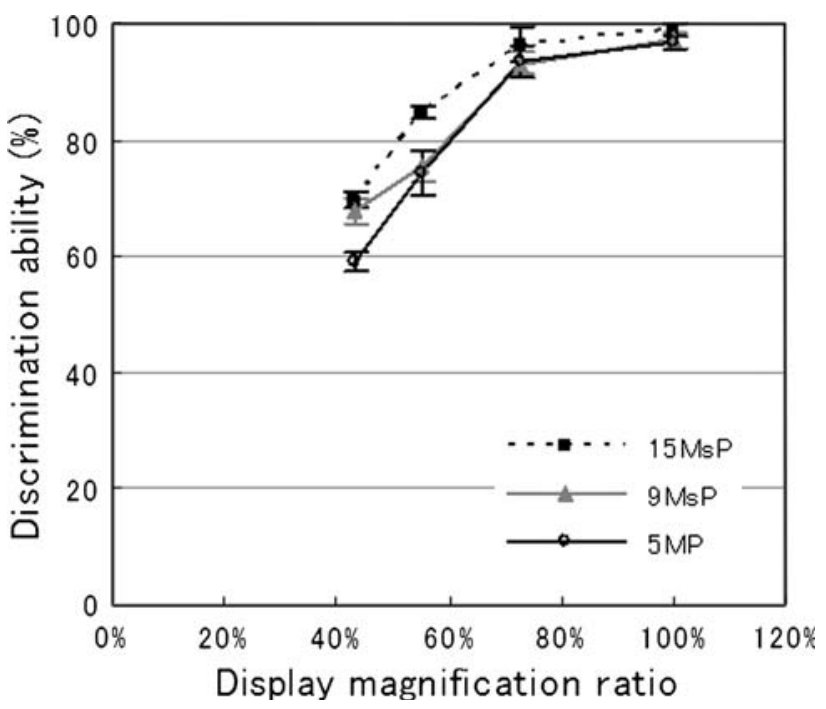

Fig. 7 Comparison of the averaged shape discrimination abilities for the three LCDs with the four types of display magnification ratio

among the three LCDs for all display magnifications examined. There were clear differences between 15-MsP and 9-MsP; the differences were statistically significant $(p<0.05)$ for all magnification ratios, in contrast to the CDMAM phantom study. Also, the detection ratio of 5-MP was lower than those for 15-MsP; these differences were statistically significant except at the $100 \%$ magnification ratio ( $p=0.0273,0.0273$, and 0.0452 , respectively).

Additionally, 9-MsP and 5-MP exhibited almost the same detection ratio except at the $100 \%$ magnification ratio, but there were no significant differences between 9-MsP and 5-MP for all magnification ratios $(p>0.0740)$.

\subsection{SSO shape study}

The averaged SSO shape discrimination ratios for the three LCDs with the four types of display magnification ratio are shown in Fig. 7, and the results of statistical analysis for each display pair are shown in Table 3. The shape discrimination ratio of $15-\mathrm{MsP}$ was markedly higher than those of the other two LCDs, and 15-MsP was significantly superior to 5-MP at all magnification ratios $(p<0.05)$. 
Table 3 Results of statistical analysis of SSO shape study for each LCD pair with the four types of display magnification ratio

\begin{tabular}{|c|c|c|c|c|}
\hline & \multicolumn{4}{|l|}{$p$-value } \\
\hline & $43 \%$ & $55 \%$ & $73 \%$ & $100 \%$ \\
\hline $15 \mathrm{MsP}$ vs. $9 \mathrm{MsP}$ & 0.4618 & $0.0350 *$ & 0.2476 & 0.0873 \\
\hline $15 \mathrm{MsP}$ vs. $5 \mathrm{MP}$ & $0.0256 *$ & $0.0278 *$ & $0.0244 *$ & $0.0325^{*}$ \\
\hline $9 \mathrm{MsP}$ vs. $5 \mathrm{MP}$ & $0.0273 *$ & 0.8335 & 0.6724 & 0.6547 \\
\hline
\end{tabular}

$* p<0.05$

Also, the difference between 15-MsP and 9-MsP was statistically significant only at the $55 \%$ magnification ratio $(p=0.0350)$.

Moreover, 9-MsP and 5-MsP showed almost the same values except at the $43 \%$ magnification ratio. The difference was statistically significant only at the $43 \%$ magnification ratio $(p=0.0273)$.

\section{Discussion}

For the CDMAM study and the SSO detection study, the superiority of 15-MsP over 5-MP in detection was clearly indicated and was not dependent on the display magnification ratio. These results showed that the substantial detectability of the SHR-LCDs was enhanced effectively with this resolution enhancement in only one (sub-pixel) direction. These two studies showed complicated results for the performance at 9-MsP. In the CDMAM study, the difference between 15-MsP and 9-MsP was not clear because of the small differences in $\mathrm{IQF}_{\text {inv }}$ results. On the other hand, in the SSO detection study, 9-MsP showed a lower detectability than did $15-\mathrm{MsP}$, and the value was almost the same as that of 5-MP. For examining the efficacy of resolution enhancement, detection studies with sufficiently small objects are more effective than CDMAM study. From this viewpoint, because the $\mathrm{IQF}_{\text {inv }}$ results are usually obtained by integrating of the results for a wide range of object sizes in the CDMAM phantom, the sensitivity of the CDMAM to the resolution difference was thought to be relatively low. Therefore, we supposed that the results of the SSO detection study were more reliable than the CDMAM study because a very small object of $0.15 \mathrm{~mm}$ was used in this study. The differences in detectability between 9-MsP and 5-MP were not significant for most of the results for both the CDMAM and the SSO detection studies. However, the $\mathrm{IQF}_{\text {inv }}$ value and the SSO detection of 9-MsP were lower than that of 5-MP only for the magnification ratio of $100 \%$. In this study, we configured the magnification ratio for 9-MsP to achieve displayed images of the same size as for the other two LCDs. Therefore, because the magnification for 9-MsP was lower than $100 \%$ (79\%), this incomplete display resulted in inferiority of $9-\mathrm{MsP}$ at the magnification ratio of $100 \%$.

At the $100 \%$ magnification ratio for the CDMAM study, although the complete image data were displayed both on 5-MP and 15-MsP, the latter provided better detectability than did the former. On the other hand, for the SSO detection study, 15-MsP was a little better than 5-MP, but their difference was not significant $(p=0.0578)$. We supposed that smoothly rendered images (i.e., less pixelized images) achieved by rendering with very fine subpixels contributed to the visibility and readability for the many objects in the CDMAM phantom.

The results of the SSO shape study clearly indicated that the shape discrimination ability of $15-\mathrm{MsP}$ was significantly better than that of 5-MP, whereas the difference at the $100 \%$ magnification ratio was meaningless because of the shape discrimination ability of more than $90 \%$ for both LCDs. The results at 9-MsP were almost the same as those at 5-MP except at the $43 \%$ magnification ratio. We considered that the superiority of $9-\mathrm{MsP}$ at the $43 \%$ magnification ratio was related to the differences in pixel (sub-pixel) number used for displaying the small objects. At the $43 \%$ magnification ratio, normal pixel numbers for a square-SSO of side $0.35 \mathrm{~mm}$ for 5-MP and 9-MsP were 2.15 and 1.7, respectively. On the other hand, the sub-pixel number for 9-MsP was 5.1. For 5-MP, the total normal pixel number to render the SSO was $2.15 \times 2.15(\approx 4.62)$, and this was not enough to render the correct shape of the SSO. In contrast, the total sub-pixel number for 9-MsP was $1.7 \times 5.1(\approx 8.67)$, and this was not too small for the SSO. Therefore, the finer sub-pixel pitch of 9-MsP improved the shape discrimination ability. On the other hand, the total sub-pixel number for 15 -MsP was $2.15 \times 6.45(\approx 13.87)$, thus, $15-\mathrm{MsP}$ showed better ability than that for 9 -MsP. In clinical mammography, shape evaluation of MCLs is important for diagnostic categorization [16, 17]. Therefore, it was expected that the good shape discrimination ability indicated in the SSO shape study would be clinically effective. The results for 9-MsP in the present study indicated that 9-MsP was at least not inferior to 5-MP. Thus, use of 9-MsP would be both advantageous and costeffective because the LCDs are produced from the same LCD panels as is 3-MP.

As the ISD technology utilizes the sub-pixels for resolution enhancement, the multi-shade-function more than 8-bit gray-scale (e.g., 11-bit gray-scale) achieved by a sub-pixel modulation, which is available in most medicalgrade displays, is consequently disabled. Thus, we performed all studies by using the 8-bit gray-scale mode. However, a previous study by Krupinski et al. [18] showed that there was no statistically significant difference between 8-bit and 11-bit gray-scales. Therefore, we 
believe that our studies using the 8-bit gray-scale mode were valid for demonstrating the usefulness of 9- and 15-MsP SHR-LCDs.

In this study, we did not compare 9-MsP with a conventional 3-MP. However, as the relation between 3-MP LCDs and 9-MsP in pixel structure is similar to that between 5-MP and 15-MsP, it was expected that 3-MP would show results inferior to those for 9-MsP, similar to the relation between 5-MP and 15-MsP demonstrated in this study.

\section{Conclusion}

We investigated the detectability and shape discrimination ability of 15- and 9-MsP SHR-LCDs with ISD technology by using a CDMAM phantom and simulated small object images.

The results clearly indicated that $15-\mathrm{MsP}$ has good potential to improve both the detectability and shape discrimination ability for small objects, such as MCLs in clinical mammograms, as compared with the conventional 5-MP. The performance of 9-MsP was not inferior to that of 5-MP throughout this study. We propose that ISD technology would be effective in improving the diagnostic accuracy of digital mammography and problems associated with the cost of expensive high-resolution monochrome displays.

\section{References}

1. Yamada T, Suzuki A, Uchiyama N, Ohuchi N, Takahashi S. Diagnostic performance of detecting breast cancer on computed radiographic (CR) mammograms: comparison of hard copy film, 3-megapixel liquid-crystal-display (LCD) monitor and 5-megapixel LCD monitor. Eur Radiol. 2008;18:2363-9.

2. Kamitani T, Yabuuchi H, Soeda H, Matsuo Y, Okafuji T, Sakai S, et al. Detection of masses and microcalcifications of breast cancer on digital mammograms: comparison among hard-copy film, 3-megapixel liquid crystal display (LCD) monitors and 5-megapixel LCD monitors: an observer performance study. Eur Radiol. 2007;17:1365-71.

3. Bacher K, Smeets P, De Hauwere A, Voet T, Duyck P, Verstraete $\mathrm{K}$, et al. Image quality performance of liquid crystal display systems: influence of display resolution, magnification and window settings on contrast-detail detection. Eur J Radiol. 2006;58:471-9.

4. Ichikawa K, Hasegawa M, Kimura N, Kawashima H, Kodera Y. A new resolution enhancement technology using the independent sub-pixel driving for the medical liquid displays. IEEE/OSA JDT. 2008;4:377-82.

5. Ichikawa K, Kodera Y, Nishi Y, Hayashi S, Hasegawa M. Development of a new resolution enhancement technology for medical liquid crystal displays. Proc SPIE. 2007;6516:65160W1-8.

6. Samei E, Poolla A, Ulissey MJ, Lewin JM. Digital mammography: comparative performance of color LCD and monochrome CRT displays. Acad Radiol. 2007;14:539-46.

7. Saunders RS, Samei E, Baker J, Delong D, Soo MS, Walsh R, et al. Comparison of LCD and CRT displays based on efficacy for digital mammography. Acad Radiol. 2006;13:1317-26.

8. Shiraishi J, Abe H, Ichikawa K, Schmidt RA, Doi K. Observer study for evaluating potential utility of a super-high-resolution LCD in the detection of clustered microcalcifications on digital mammograms. J Digit Imaging. 2009 [Epub ahead of print].

9. Fan J, Roehrig H, Sundareshan MK, Krupinski E, Dallas WJ, Gandhi K. Evaluation of and compensation for spatial noise of LCDs in medical applications. Med Phys. 2005;32:578-87.

10. Badano A, Gagne RM, Jennings RJ, Drilling SE, Imhoff BR, Muka E. Noise in flat-panel displays with subpixel structure. Med Phys. 2004;31:715-23.

11. National Electrical Manufacturers Association. Digital Imaging and Communications in Medicine (DICOM), Part 14: Grayscale Standard Display Function PS 3.14, 2007.

12. Samei E, Badano A, Chakraborty D, Compton K, Cornelius C, Corrigan K, et al. Assessment of display performance for medical imaging systems: executive summary of AAPM TG18 report. Med Phys. 2005;32:1205-25.

13. Rivetti S, Lanconelli N, Campanini R, Bertolini M, Borasi G, Nitrosi A, et al. Comparison of different commercial FFDM units by means of physical characterization and contrast-detail analysis. Med Phys. 2006;33:4198-209.

14. Thomas JA, Chakrabarti K, Kaczmarek R, Romanyukha A. Contrast-detail phantom scoring methodology. Med Phys. 2005;32:807-14.

15. Bijkerk KR, Thijssen MAO, Arnoldussen ThJM. Manual CDMAM-phantom type 3.4. St. Radboud: University Medical Center Nijmegan; 2001.

16. Park JM, Choi HK, Bae SJ, Lee MS, Ahn SH, Gong G. Clustering of breast microcalcifications: revisited. Clin Radiol. 2000; $55: 114-8$.

17. Lafontan de B, Daures JP, Salicru B, Eynius F, Mihura J, Rouanet $\mathrm{P}$, et al. Isolated clustered microcalcifications: diagnostic value of mammography—series of 400 cases with surgical verification. Radiology. 1994;190:479-83.

18. Krupinski EA, Siddiqui K, Siegel E, Shrestha R, Grant E, Roehrig H, et al. Influence of 8-bit vs 11-bit digital displays on observer performance and visual search: a multi-center evaluation. J Soc Inf Disp. 2007;15:385-90. 